equally essential, radiation is better controlled. Further radiation protection is provided by incorporating a radiationproof shield in the back of the cassette holder, which will always absorb the main, central $x$-ray beam during any examination.

Only four kilovoltage settings (perhaps three, or, maybe, five-there is room for negotiation) are required: the almost universal stepless control from 40 to $130 \mathrm{kV}$ is a luxury not in any way dictated by necessity. Some units use an anatomical exposure chart: a pointer or a lever is set against the drawing of the required part of the body and the correct exposure produced, with a positive or negative addition for the large or small patient. Radiologists and radiographers who care to consider this apparently frightening limitation and who then experiment for themselves will find that for the common examinations this range of exposures, particularly a limited $\mathrm{kV}$ setting, is entirely sufficient.

Maintenance has also been simplified: the $x$-ray generators must be of modular construction and so designed that replacement of one module does not require recalibration. In future much of these sets can be manufactured in developing countries and only the tubes, cables, and some of the electronic parts will need to be imported. Film processing is likely to be by hand and the group recognised that in hot and cold countries temperature control is difficult. The DuPont Company, of Wilmington, Delaware, have, however, devised a method of processing which uses an additive that can be mixed with almost any $x$-ray-film developer and will permit time-temperature processing from $20^{\circ}$ to $35^{\circ} \mathrm{C}$ so that solutions will not need to be cooled. A simple time-controlled method of processing can easily be taught.

These Basic Radiological System units are now on trial in various parts of the world and four or five different commercial units will soon become available. In the rural or cottage hospital any nurse, orderly, pharmacist-or even a doctorcan be taught to use the unit with the aid of a manual of radiographic technique, already on trial, which uses a pictorial step-by-step sequence. The Basic Radiological System Advisory Group has concentrated on about 80 routine radiographic projections, which will supply the needs of almost every peripheral hospital.

The advisory group has had to face one other problem. Most medical students are taught elaborate, complex radiology. Aspiring physicians are asked to interpret barium-meal pictures, cardiac or vascular studies, or recognise bone tumours. These will be the province of specialists, not the concern of $99^{\circ}$ o of their patients. The advisory group is therefore attempting (a word used advisedly) to design a diagnostic handbook to match the radiographs produced by its machines. Knowing what to look for and where may be the most important part of radiodiagnosis, as it is with many other aspects of physical examination.

No one need feel threatened by the Basic Radiological System concept. Radiology cannot be a closed shop and especially if there are no radiologists. There will never be enough specialist radiologists anywhere: in developing countries there is sometimes one radiologist for one million people. In the circumstances for which the Basic Radiological System unit is designed little radiological support can be expected, but specialist radiologists should visit the hospitals, look at difficult cases, and discuss problems with local practitioners if standards are to be maintained. There is more than enough work for radiologists in the modern, complex radiographic procedures and in the whole topic of imaging with its technical advances: the Basic Radiological System at the periphery will probably free them to do this type of work more satisfactorily and is likely to stimulate more of the difficult procedures rather than reduce the overall work for all concerned.

Professor of Diagnostic Radiology,

P E S PALMER

University of California,

Davis 95616

${ }^{1}$ Chamberlain $\mathrm{RH}$. Basic radiology. A worldwide challenge. $\mathcal{F} A M A 1970$ 214:1687-92.

2 Cockshott WP. Diagnostic radiology: geography of a high technology. AFR 1979;132:339-44.

${ }^{3}$ Palmer PES. Radiology in basic-care hospitals and clinics. In: Approaches to planning and design of health care facilities in developing areas. Vol 3. Geneva: World Health Organisation, 1979:83-124.

- Racoveanu NT. The basic radiological unit: a new concept for increasing radiodiagnostic population coverage. Alexandria: World Health Organisation, 1978. (WHO document EM/RC28/9.)

\section{Fetal ascites and congenital heart disease}

As a result of the decrease in both the incidence and the severity of rhesus haemolytic disease hydrops fetalis has become less common. Non-immunological causes of hydrops, however, are well recognised. Driscoll' has listed over 25 associations, including alpha-thalassaemia; twin transfusion syndrome; pulmonary lymphangiectasia; achondroplasia; maternal diabetes; and infections such as toxoplasmosis, cytomegalovirus, and syphilis, as well as cardiac and multiple congenital abnormalities. Cardiac abnormalities appear to be particularly common; and in a series of 42 infants who died with pleural effusions, ascites, and generalised oedema eight had cardiac anomalies. ${ }^{2}$ The type of anomaly varied and most were associated with non-cardiovascular anomalies.

Although the incidence of hydrops fetalis is declining, fetal ascites is now diagnosed much more often. Ascites occurs for reasons similar to hydrops and may often represent an earlier stage of the same condition. Routine ultrasound scanning in pregnancy gives the chance of earlier diagnosis. Fetal ascites is most easily recognised from a transverse scan of the abdomen, the accumulated fluid presenting as an area that gives no echoes, usually crescentic in outline, between the abdominal wall and the viscera. ${ }^{3}$ In severe cases the abdominal contents are compressed backwards and upwards and are reduced to a small knot of tissue floating in a large volume of ascitic fluid. If the abdominal circumference is measured it will naturally be increased in proportion to the amount of free fluid present. Obviously an attempt should be made to detect any other anomalies. In a recent case from Paris ${ }^{4}$ ultrasound examination was carried out at 24 weeks because fetal movements were absent. The most striking feature of the sonogram was very large ascites, which resulted in a clear picture of the various viscera. The liver was midline and appeared to float in ascitic fluid and the intestinal loops were displaced along the whole length of the vertebral column; while on further examination the cardiac area was seen to have no intraventricular septum. A medical abortion was carried out on the basis of the ultrasonic findings and necropsy confirmed a major malformation of the heart, which had a single ventricle. This case clearly shows that the ultrasonic diagnosis of fetal ascites may suggest a cardiac abnormality even in the middle trimester of pregnancy, when termination may be considered. 
Many will find a more important lesson in a case report from King's College Hospital." A scan at 28 weeks showed fetal ascites and cardiac enlargement. When the scan was repeated at 34 weeks ascites was still present and the heart showed gross dilatation of the left atrium and left ventricular hypertrophy. The internal diameter of the aortic root, measured by real-time scanning, was less than one-third of normal. At delivery two weeks later the baby showed initial cyanosis and cardiac failure within 24 hours, dying despite supportive measures. Necropsy confirmed a small aortic root with left ventricular hypertrophy. The baby was otherwise normal. The case is unusual in that fetal ascites was due to a single cardiac anomaly causing cardiac failure. The authors postulate that the aortic root stenosis had increased left ventricular pressure and caused hypertrophy. The hypertrophy and subendocardial fibroclastosis increased left ventricular stiffness, leading to left atrial hypertension and dilatation. This in turn reduced the flow from the right to left atrium through the foramen ovale. Consequently the cardiac output was maintained predominantly by the right ventricle, and volume overloading produced right atrial and right ventricular dilatation, with subsequent cardiac failure and ascites.

When fetal ascites is seen on ultrasound examination an associated cardiac defect should be suspected. Further experience and development of the technique may enable a remediable defect to be seen. Even now, when ascites is shown and is apparently idiopathic the baby should if possible be delivered in a hospital where immediate cardiac assessment will permit early surgery in suitable cases.

Consultant Obstetrician and Gynaccologist,

J G FEeNEY

Roval Infirmary, Huddersfield HD3 3EA

Driscoll SG. Hydrops fetalis. N Engl f Med 1966;275:1432-4.

Beischer NA, Fortune 1)W, Macafee J. Nonimmunologic hydrops fetalis and congenital abnormalities. ()bstet Gynecol 1971;38:86-95.

Law RG. Ultrasound in clinical obstetrics. Bristol: John Wright, 1980.

1 Henrion R, Aubrey JP. Fetal cardiac abnormality and real-time ultrasound study: a case of Ivemark svndrome. Contrib Gynecol Obstet 1979; $6: 119-22$.

Allan L, Little 1), Campbell S, Whitehead MI. Fetal ascites associated with congenital heart discase. Case report. Brf Obstet Gynaecol 1981; $88: 453-5$.

\section{Acute viral encephalitis}

Acute viral encephalitis poses difficulties both in the very real problems of diagnosis and management and in complexities of definition and categorisation. Even when the diagnosis has been made or assumed, comparison between the severity of individual cases is very difficult. One possibility might be a scoring system, with points being allotted for various features: one for neck stiffness, one for fever, two for cranial nerve abnormalities, three for altered consciousness, four for coma, and so on. The total score for an individual patient would then give some idea of the severity of the illness and would facilitate comparison of cases. Wayne introduced a points system of this kind in the diagnosis of thyroid disease. ${ }^{1}$ Such a categorisation, however, begs the question, for it depends on the certain identification of virus infections and so-called postviral encephalitis.

At the bedside, then, the immediate problem is one of diagnosis. The clinician has to work his way through a whole range of acute or subacute neurological disorders with a definite aetiology. The common forms of purulent meningitis do not usually cause difficulties, but the differential diagnosis must take account of tuberculous meningitis, and despite the most refined laboratory techniques in some cases a trial of antituberculosis chemotherapy will be justified. Cerebral abscess, tumour, infarct, or haemorrhage all have to be excluded. Among the conditions that cause a lymphocytosis in the cerebrospinal fluid are syphilis, fungal meningitis, leptospirosis, brucellosis, Behçet's disease, cerebral sarcoidosis, and acute demyelinating disorders.

The initial diagnostic objective is identification of any condition which demands urgent treatment; in practice the possibilities that cause real and common anxiety are tuberculosis, abscess, tumour, and perhaps some rare forms of meningitis. In one series ${ }^{2}$ of 120 patients suspected of having viral encephalitis seen in Glasgow over a four-year period nearly half proved to have diseases other than encephalitis.

A more recent series of 60 patients from the London Hospital $^{3}$ included 12 patients with known viral infection, 29 with acute viral infection of undetermined type, and 19 patients in whom an encephalitic illness followed a viral infection (postinfective encephalitis). The patients with postinfective encephalitis had the best prognosis while those with herpes simplex virus encephalitis had the worst, with twothirds dying. In England and Wales each year about 175 deaths are attributed to encephalitis, of which perhaps 20 are proved virologically, ${ }^{4}$ and herpes simplex virus has acquired a deserved reputation for high morbidity and mortality: untreated the mortality rate may be as high as $75^{\circ},{ }^{4}$

The clinical dilemma is most acute in those patients in whom a diagnosis of herpes simplex virus encephalitis is likely on the grounds of history, clinical examination, and the results of readily accessible laboratory tests but in whom the diagnosis has not been confirmed. Confirmation requires brain biopsy, but treatment should be undertaken as soon as possible; how soon, then, should brain biopsy be carried out ? A simple, rapid, reliable, and preferably non-invasive method to confirm the diagnosis of herpes simplex virus encephalitis is urgently needed. The alternatives to brain biopsy are at best only indirect and probably non-specific. In patients with acute viral encephalitis the electroencephalogram may show a generalised cerebral disorder with bilateral slowing in the delta and theta frequencies; in herpes simplex virus the electroencephalogram changes may be focal, but unfortunately they do not seem to be pathognomonic and probably merely reflect the severity of focal changes." Air encephalography and cerebral angiography may occasionally disclose a lesion in a patient who is eventually shown to be suffering from herpes simplex virus encephalitis, but these techniques are seldom indicated when computed tomography is available. Computed tomography has shown abnormalities in at least two series of patients, ${ }^{67}$ and in two of the three patients in the recent London Hospital series with herpes simplex virus encephalitis scanning showed decreased attenuation on one temporal lobe in one case and in a parietal lobe in another. ${ }^{3}$ These abnormalities occur early in the illness and come close to providing specific diagnosis without histopathological or viral confirmation.

The results of treatment of encephalitis are unpredictablewhich is scarcely surprising in a condition as variable as acute viral encephalitis and including at least two pathological subgroups (viral and postinfective) in which diagnostic techniques leave a lot to be desired. In herpes simplex virus encephalitis the main approaches to treatment are to relieve the cerebral oedema induced by the inflammatory response to the virus, and to try to stop virus replication. Surgical decompression, ${ }^{8}$ dexamethasone, ${ }^{9}$ and mannitol ${ }^{10}$ have all been used in attempts to control cerebral oedema, but the results have been incon- 